\title{
Étalement urbain et consommation d'espace. Étude comparée de Besançon, Belfort et Montbéliard
}

Urban sprawl and consumption of space. A comparative study of Besançon, Belfort and Montbéliard

Flächenhafte Expansion und Zersiedlung prägen mittlerweile die Entwicklung

aller französischen Städte

Jean-Philippe Antoni et Samy Youssoufi

\section{(2) OpenEdition \\ Journals}

Édition électronique

URL : http://journals.openedition.org/rge/1433

DOI : $10.4000 /$ rge. 1433

ISSN : 2108-6478

Éditeur

Association des géographes de l'Est

Édition imprimée

Date de publication : 1 juin 2007

ISSN : 0035-3213

Référence électronique

Jean-Philippe Antoni et Samy Youssoufi, «Étalement urbain et consommation d'espace. Étude comparée de Besançon, Belfort et Montbéliard », Revue Géographique de l'Est [En ligne], vol. 47 / 3 2007, mis en ligne le 18 octobre 2011, consulté le 10 décembre 2020. URL : http:// journals.openedition.org/rge/1433; DOI : https://doi.org/10.4000/rge.1433

Ce document a été généré automatiquement le 10 décembre 2020.

Tous droits réservés 


\title{
Étalement urbain et consommation d'espace. Étude comparée de Besançon, Belfort et Montbéliard
}

\author{
Urban sprawl and consumption of space. A comparative study of Besançon, \\ Belfort and Montbéliard \\ Flächenhafte Expansion und Zersiedlung prägen mittlerweile die Entwicklung \\ aller französischen Städte
}

Jean-Philippe Antoni et Samy Youssoufi

1 Depuis la fin de la seconde Guerre Mondiale, toutes les études liées aux phénomènes d'urbanisation et de périurbanisation montrent que l'étalement urbain s'est généralisé à l'ensemble des agglomérations françaises, de grande, de moyenne et même de petite taille (Le Jeannic, 1997). L'accroissement spatial des aires urbaines, qui va de pair avec un éclatement des lieux de vie entre les centres anciens et les franges périurbaines constitue dès lors une nouvelle donne qu'il s'agit de prendre en compte et de gérer. Cette gestion nécessite cependant de comprendre les tenants et les aboutissants de l'étalement urbain, de saisir les enjeux et les conséquences qu'il provoque dans le court, le moyen et le long terme, tant sur le plan spatial que social et environnemental (Barcelo, 1993) : l'étalement urbain ne doit pas se considérer comme un problème en lui-même ; il apparaît plutôt comme cas particulier du processus général d'urbanisation, et se caractérise par une morphogenèse urbaine particulière, associée à une construction spécifique, possiblement ségrégative et peu durable de la ville contemporaine (Antoni, 2003). La sur-consommation d'espace qu'il induit, et sur laquelle nous nous concentrons ici, stigmatise une partie de ces problèmes.

\section{Etalement urbain et consommation d'espace}

2 Pour comprendre l'étalement urbain, il importe de ne pas le considérer comme un processus isolé ou orphelin apparu avec la fin des Trente glorieuses, mais a contrario 
de l'envisager à travers ses causes et ses conséquences, qui relèvent quant à elles de la nature même des processus de métropolisation (Bassand, 2000). L'étalement urbain s'inscrit alors dans un schéma synthétique, montrant que l'enchevêtrement des processus à l'œuvre conduit naturellement à une consommation toujours plus importante d'espace par les aires urbaines.

\section{A. L'étalement urbain}

3 La métropolisation correspond à un mouvement important de concentration de la population dans les métropoles, i.e. dans les grandes villes qui organisent un territoire plus ou moins vaste. Elle provoque la densification des activités, en même temps que des populations, autour des centres urbains qui procurent le maximum d'aménités spécifiques, évaluées tant sous l'angle des emplois, que des services et de plus en plus, sous celui de la diversité des possibilités de loisir. En conséquence, les villes connaissent aujourd'hui une expansion spatiale dont la vitesse est sans précédent dans l'histoire urbaine : la macroforme que dessine la surface bâtie occupée par les territoires urbains croît de manière inexorable et uniforme, autorisant d'autant à assimiler l'étalement urbain à un processus tentaculaire, pour lequel les références au monde biologique (métaphore organiciste) sont de bonnes images (Monnet, 1997).

4 À l'échelle intra-urbaine, l'image du Blob ${ }^{1}$ que renvoie cette croissance importante des espaces urbains se révèle toutefois plus subtile. Les villes ne croissent pas de manière régulière autour d'un rayon tracé en ligne droite qui relie leur centre et leurs périphéries les plus éloignées. Au contraire, il existe de nombreuses ruptures le long de cette ligne souvent très accidentée et complexe, dont on peut mesurer la dimension fractale (Frankhauser, 1994). Ces ruptures affectent autant les caractéristiques physiques des zones bâties (les bâtiments du centre ancien ne sont pas du même type que ceux de la périphérie résidentielle et pavillonnaire à la frange des bois et des champs) que les caractéristiques socio-professionnelles des populations qui les habitent (le centre, les périphéries et les transects qui les composent font l'objet d'enjeux spécifiques suivant la catégorie socio-professionnelle, voire socio-ethnique des populations qui y vivent). L'organisation radioconcentrique qui résulte de ces enjeux a été particulièrement mise en évidence dès les années 1930 par les sociologues de l'école de Chicago (Grafmeyer, 1979), et les études des géographes et des démographes d'aujourd'hui continuent d'en montrer l'actualité. Ainsi, si l'on suit la logique de ce schéma, il existe un lien nécessaire entre trois éléments qui caractérisent autant d'aspects complémentaires du processus d'étalement au sein des aires urbaines :

5 1. Les activités économiques (industrielles et tertiaires) se concentrent à l'intérieur des aires urbaines et contribuent à en augmenter l'attractivité et à la compétitivité. Cette croissance, que la densité pousse naturellement hors des centres, s'accompagne d'une pression et d'une promotion immobilières très rentable dans les zones périphériques ;

6 2. La population, dont la croissance accompagne la localisation des activités industrielles et tertiaires, se localise également en périphérie, selon un compromis entre la localisation des lieux de travail et les critères qui répondent à la qualité de vie recherchée (ou proposée par les promoteurs) en terme de logement et de voisinage. L'ensemble s'ajuste au prix de déplacements domicile-travail d'une longueur et d'une durée croissantes ; 
7 3. Les surfaces bâties, qui accueillent à la fois des activités économiques nouvelles et des populations, augmentent. Les périphéries sont privilégiées, soit par le monde industriel (parce que la plus grande liberté de construction y offre une réponse aux exigences fonctionnelles et logistiques des activités d'aujourd'hui), soit par les catégories qui souhaitent accéder à la propriété d'une maison individuelle (parce qu'elles bénéficient d'une proximité quasi-identique aux aménités urbaines et rurales).

\section{B. La consommation d'espace}

Le schéma présenté ci-dessus est classique, voire théorique, il induit pourtant l'idée que la consommation d'espace par les aires urbaines va croissante. Partant de ce constat, on peut poser l'hypothèse qu'une fois rapportée à leur population, la surface bâtie des aires urbaines actuelles est nécessairement plus importante que celle des villes d'hier, et que l'écart dans ce sens va croissant. Qu'est-ce alors que la consommation d'espace? L'expression est rarement référencée dans les dictionnaires de géographie ${ }^{2}$. Intuitivement, on pourrait l'entendre selon deux acceptions : celle de «faire usage de », et celle de «mener à son terme ». Dans le premier cas de la consommation d'espace par la ville, l'expression pourrait donc s'associer à l'idée qu'elle utilise l'espace périphérique, qu'elle s'en sert pour fonctionner. Dans le deuxième cas, elle le mène à son terme, dans le sens ou elle l'achève (ou l'absorbe) et lui confère un état terminal qui n'évolue plus : une fois devenu urbain, l'espace ne connaît plus de point de retour. Dans le dictionnaire dirigé par G. Wackermann, la notion apparaît effectivement « imagée " et induit l'idée que « l'espace n'a pas été conservé en l'état, mais [a été] soumis à des modifications, des perturbations, qui réduisent l'espace disponible, quantitativement et qualitativement $»^{3}$. Trois observations semblent corroborer cette idée que, dans le cadre de l'étalement urbain, la consommation d'espace va croissante :

9 1. Une fois relayée en périphérie et libérée des contraintes de la densité du centre-ville, la construction des bâtiments accueillant les zones d'activités (entrepôts, industries, etc.) a désormais l'habitude de " prendre ses aises " : l'espace disponible et consommable fait partie des atouts logistiques intrinsèques aux périphéries urbaines (Merenne-Schoumaker, 2002, 2003) ;

10 2. La construction de logements est majoritairement orientée vers les maisons individuelles adaptées aux mobilités de l'automobile. D'une part, on sait que la densité plus faible des maisons individuelles entraîne une consommation d'espace plus importante que les logements collectifs et denses des zones centrales. D'autre part, lorsqu'elles sont groupées en lotissements, elles s'imposent souvent comme des excroissances « en fin de réseau » au sein du bâti urbain, dont la connectivité et les relations avec les constructions à venir posent la question de l'optimisation fonctionnelle, qui n'est souvent rendue possible que par le gel d'espaces tampons, soit une nouvelle consommation d'espace (dans la mesure où le caractère résiduel de ces espaces n'en permet pas une utilisation optimale);

11 3. À cela s'ajoute la présence des équipements accompagnant les constructions, essentiellement résidentielles, dans les périphéries des villes (stades, parcs, squares, aires de jeux, etc.), dont on peut montrer que l'importance relative va également croissante dans les zones périurbaines, répondant à un choix d'habiter et à une exigence en terme de qualité de vie, qui sont devenus prégnants (Antoni, 2003). 
évidemment à être confrontée aux réalités et aux spécificités du terrain. Le rapport entre la population et la surface bâtie reste-t-il proportionnel au cours du temps (cas pour lequel la consommation d'espace est linéaire), ou bien connaît-il un effet multiplicateur (cas pour lequel il y a bien sur-consommation entre un temps $t$ et un temps $t+1)$. En Franche-Comté, par exemple, quid du processus d'étalement urbain? Retrouve-t-on ici les caractéristiques que les études à l'échelle nationale nous permettent d'attendre ? Observe-t-on une progression de la consommation d'espace ou, au contraire, reste-t-elle plus ou moins proportionnelle à sa croissance démographique? Originales par leurs dimensions industrielle, économique ou géographique (cf. II), les aires urbaines franc-comtoises offrent ici un terrain d'étude privilégié dont il convient de décrire quelques-unes des spécificités pour mieux en saisir le contexte.

\section{Les villes de Franche-Comté}

16 La Franche-Comté se caractérise par une structure urbaine très différente de celle des régions qui l'entourent ${ }^{4}$. Le nombre de grandes agglomérations y apparaît en effet limité. On ne compte par exemple que 24 villes de plus de 5000 habitants (soit 1,34\% de l'ensemble des communes franc-comtoises contre 5,05 \% à l'échelle de la France). Néanmoins, parmi celles-ci, trois agglomérations sortent nettement du lot par l'importance relative de leur population : Besançon, Belfort et Montbéliard (Figure 1). Que ce soit par leur situation géographique, leurs structures économiques et sociales ou les aléas historiques auxquels elles ont été confrontées, ces trois villes ont connu des évolutions assez différentes : alors que Besançon connaît un gain de population, la situation est plus problématique dans le Nord-Franche-Comté où Belfort semble stagner tandis que Montbéliard perd de la population (Floch, 2000 ; Moine, 2001 ; Dussaud, 2007). Dès lors, il semble pertinent de décrire ces évolutions en lien avec la consommation d'espace. 
Figure 1 : En Franche-Comté, trois agglomérations se distinguent par l'importance de leur population : Besançon, Belfort et Montbéliard, qui ont chacune connu des évolutions démographiques, industrielles et spatiales très différentes.

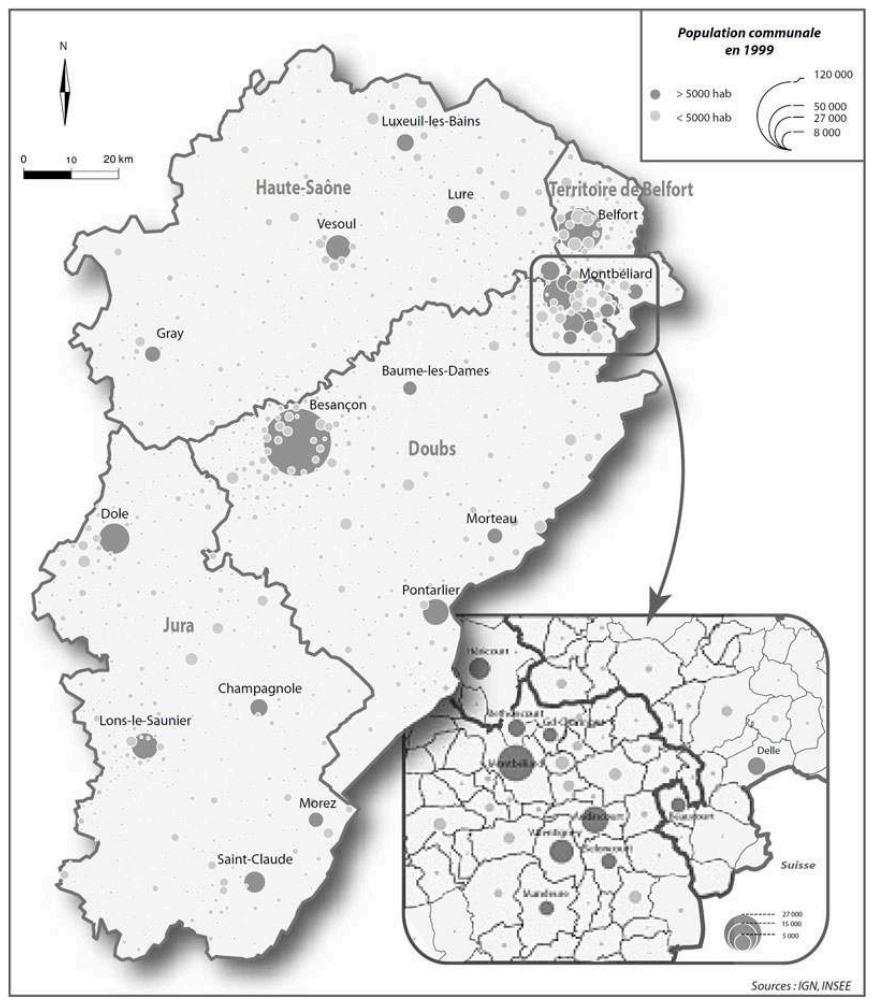

\section{A. Besançon, capitale régionale}

Besançon est l'une des plus petites capitales régionales de France. Elle compte environ 117000 habitants dans la ville même et près de 170000 dans l'agglomération ${ }^{5}$. Elle occupe un site exceptionnel à l'intérieur d'un méandre du Doubs presque recoupé, choisi au départ pour ses qualités défensives. Les quartiers centraux très denses à l'intérieur de « la boucle » en ont hérité. Longtemps, Besançon est pourtant restée une ville de taille modeste : 55000 habitants au début du siècle, moins de 65000 à l'issue de la seconde Guerre Mondiale. Ville bourgeoise, plutôt conservatrice, c'est alors l'exemple typique d'une cité de province qui ne bénéficie du ressort d'aucune activité majeure, si ce n'est l'administration d'un département et d'une université modeste (« admirée par les siens plus que par les autres » selon A. Fremont, 2003).

Jusqu'en 1945, l'horlogerie implantée au cours de la Révolution constitue son unique spécialisation industrielle et emploie jusqu'à 8000 personnes dans la ville et quelquesuns de ses faubourgs. Après la Seconde Guerre mondiale par contre, l'agglomération va considérablement se développer. Tout d'abord, Besançon devient capitale administrative par la régionalisation. Parallèlement, l'industrie bisontine se diversifie et augmente en qualité, poussée par la croissance des années 1960 qui bénéficie de l'implantation ou de l'extension de grosses unités de production (Rhône-Poulenc, Lip, Kelton, Timex...). Mais celles-ci disparaissent ou diminuent leurs effectifs pendant la crise des années 1970. Sur le plan de l'urbanisation, la concentration autour du centreville a été très forte, mais depuis, ce sont les zones périurbaines qui tirent la croissance de l'agglomération. Elles ont toutefois défini une nouvelle donne, que l'horlogerie et 
des activités de haute précision (couplées avec les recherches des laboratoires universitaires ou privés) illustrent.

Besançon se revendique aujourd'hui capitale de l'horlogerie (environ 3000 emplois) et plus généralement des microtechniques. Afin d'accueillir ces activités nouvelles, la ville s'est doublée d'une périphérie de zones industrielles, qui intègre notamment un campus scientifique, ainsi que des logements, d'abord sous la forme de grands ensembles de type open planning (quartier Planoise), puis de maisons individuelles au contact des zones "naturelles ». Compte tenu de la topographie du site initial, le tout s'est particulièrement développé au nord de la boucle du Doubs, le long d'une sorte d'axe tangentiel au noyau ancien, orienté vers Belfort au nord-est et vers Dole, puis Dijon au sud-ouest. Ainsi, une large bande périurbaine ceinture désormais la ville dans un environnement de grande qualité paysagère ${ }^{6}$.

\section{B. Belfort}

Aux portes de l'Alsace, l'ensemble urbain de Belfort-Montbéliard compte un peu moins de 300000 habitants et constitue la principale aire urbaine de la région, loin devant Besançon. Toutefois, cet ensemble apparaît disparate et s'assimile aisément à un archipel ou à une constellation de bourgs peu peuplés : Belfort, la plus grande ville, dépasse à peine 50000 habitants alors que Montbéliard en compte moins de 30000 . L'aire urbaine de Belfort-Montbéliard n'échappe toutefois pas aux risques et aux travers de la mono-industrie, tant à Belfort autour d'Alstom qu'à Sochaux autour de Peugeot.

21 À Belfort, le lion de Bartholdi, érigé en mémoire de la résistance que les soldats français opposèrent à l'offensive allemande de 1870 , suffit à témoigner du caractère militaire d'une ville garnison longtemps figée dans cette unique fonction. Belfort reste française à l'heure où l'Alsace devient allemande, et se doit d'accueillir la préfecture de son territoire (le Territoire de Belfort), qui devient le plus petit département de France. Parallèlement, les entrepreneurs alsaciens, qui souhaitent demeurer sous administration française, y établissent de nouveaux sièges et se spécialisent dans l'équipement électrique lourd et la fabrication de locomotives. La réussite économique spectaculaire des deux entreprises Alstom et Dollfus-Mieg modèlent alors très largement la nouvelle physionomie de la ville: «une forteresse gigantesque mais obsolète, une préfecture en miniature, une grand cité industrielle » (A. Frémont, 2003).

Dollfus-Mieg, puis Bull ont désormais disparu de l'horizon belfortain. Dans l'aire urbaine belfortaine ${ }^{7}$, l'entreprise Alstom reste le principal employeur (l'entreprise fournit près du quart de l'emploi privé salarié de l'aire urbaine) et sa santé économique, comme sa stratégie de production, commande partiellement l'évolution de la population. Ainsi, jusqu'en 1975, le recours à une main d'œuvre venue du monde rural de Haute-Saône ou immigrée était de mise, mais il a été stoppé net par la crise économique et la robotisation, qui ont entraîné une baisse des effectifs industriels jusqu'en 1990 (Laurent, 2000). L'aire urbaine connaît alors une perte croissante de population (260 départs nets par an entre 1975 et 1982, puis 300 entre 1982 et 1990 ; l'ensemble correspondant à une perte d'effectifs de 130 emplois par an chez Alstom). Aujourd'hui, la population augmente toutefois à nouveau, appuyée sur un tertiaire en développement et sur l'essor du pôle universitaire Belfort-Montbéliard. Dans le même 
temps, la périurbanisation se poursuit en privilégiant six axes d'expansion spatiale, de part et d'autre des axes routiers les plus importants.

\section{Montbéliard}

23 À une quinzaine de kilomètres au sud, le lion de Montbéliard symbolise plus encore la réussite industrielle du Nord de la Franche-Comté, à travers le site du constructeur automobile Peugeot, l'un des plus importants d'Europe ${ }^{8}$. Aux périphéries de la ville, c'est toute une nébuleuse de " gros villages » qui accompagne cet essor comme autant de cités de résidence (genre cités dortoirs) ou des centres de sous-traitance (Héricourt, Béthoncourt, Audincourt, Valentigney, etc.) dont la configuration spatiale est un héritage du XVIIIe siècle, reprenant la localisation des forges, des moulins et des petites usines métallurgiques et textiles qui ont employé les ouvriers issus du premier exode rural. Dans le prolongement de la tradition locale, la mono-industrie Peugeot s'insère dans une nébuleuse urbaine qui poursuit l'organisation de l'espace industriel du milieu rural ${ }^{9}$.

Cependant, la situation démographique de Montbéliard diffère largement des deux précédents exemples : la ville fait partie des rares aires urbaines françaises de plus de 100000 habitants qui connaissent une baisse démographique. Entre les recensements de 1962 et 1982, la ville est pourtant portée par les Trente glorieuses et connaît une croissance assez soutenue, du moins plus rapide que l'ensemble des aires urbaines de France. La démographie culmine en 1982 (192 000 habitants). La crise économique et le changement de méthode de travail vont alors provoquer une chute importante de l'emploi, qui se traduit par des départs en pré-retraite et une incitation au retour au pays pour les populations issues des vagues immigrées italienne (arrivée dans les années 1950) ou maghrébine (arrivée dans les années 1970) : 5000 immigrés partent en 1985 et, de 1975 à 1990, on enregistre un déficit migratoire de 1800 personnes, tout juste compensé par l'excédent naturel (Laurent, 2000). Depuis 1990, le déficit migratoire s'est réduit à 1200 personnes par an, grâce aux emplois tertiaires nouvellement créés et à l'université qui garde désormais captive une population d'étudiants qui, jusque-là, rejoignait les universités de Strasbourg ou de Besançon. Seules trois communes dégagent un excédent migratoire entre 1990 et 1999: Taillecourt, Sainte-Suzanne et Seloncourt, dans lesquelles les zones pavillonnaires continuent de se développer (quartiers de Berne et du Criolet à Seloncourt par exemple). Ailleurs, la crise économique qui touche l'industrie automobile se répercute sur la quasi-totalité des quartiers.

\section{Mesurer la consommation d'espace dans les aires urbaines comtoises}

Pour étudier la consommation d'espace dans les trois principales agglomérations de Franche-Comté, les évolutions démographiques fournissent de manière relativement précise la population. Mais il demeure nécessaire de collecter les données permettant de calculer leur surface bâtie. Ces données sont bien sûr des informations spatiales ; mais ce sont aussi des informations temporelles qui doivent montrer comment les choses ont changé, à savoir ici, comment l'espace a été construit - ou consommé - au cours du temps. 


\section{A. Le problème des données : des contraintes spatio-temporelles} démographique décrite plus haut pose essentiellement deux problèmes. D'une part, aucune source de données ne permet a priori de saisir un processus : elles n'en offrent que des images statiques. D'autre part, ces images statiques sont relevées à un certain rythme qui n'est que rarement (et c'est alors un hasard), celui du processus étudié. L'acquisition des données permettant de mesurer l'étalement relève alors dans certains cas d'une véritable chasse au trésor. En effet, les documents cartographiques (cartes topographiques, photographies aériennes, images satellites) montrant l'évolution d'une ville depuis les années 1950-1960 ont souvent disparus des mairies et des agences d'urbanisme (leur mise à jour constitue d'ailleurs souvent un réel enjeu de gestion de l'information géographique). L'IGN quant à lui, ne commercialise plus ses productions anciennes et actualisées depuis. Un recours aux archives (municipales dans le cas de Belfort, universitaires du laboratoire de géographie dans le cas de Besançon) a toutefois permis de géoréférencer d'anciennes cartes pour les étudier. À Montbéliard par contre, une recherche moins fructueuse nous a conduit à utiliser un jeu de données différent mais décrivant également l'état de la tache urbaine dans le temps (mais à des dates différentes des précédents documents) : les fichiers numérisés du cadastre (DGI). De ce fait, il importe de décrire ces données disparates et mobilisables, afin d'identifier les biais que comporte leur comparaison pour l'étude de l'étalement urbain et de la consommation d'espace. À Besançon, les cartes topographiques au 1/25 000 produites par l'IGN offrent une image de la ville et de ses périphéries dans les années 1960, 1980 et 2000. Elles permettent de suivre avec précision l'évolution des surfaces bâties sur 59 communes autour de Besançon, en identifiant les bâtiments et les maisons individuelles construits dans chacune des deux périodes. À Belfort, un jeu de cartes similaire montre la ville et ses environs en 1955, 1975 et 1995, soit une étendue globalement comparable à la précédente, pour une surface comprenant 29 communes. À Montbéliard par contre, les fichiers informatiques de la Direction Générale des Impôts ${ }^{10} \mathrm{mis}$ à notre disposition pour identifier les maisons et bâtiments construits pour l'ensemble de la Communauté d'Agglomération du Pays de Montbéliard (soit 29 communes) ne couvrent que trois dates particulières : 1978, 1990, 2002. Ces trois jeux de données permettent globalement de décrire l'urbanisation de manière diachronique ${ }^{11}$.

Cependant, disposer de ces informations ne suffit pas ; afin de visualiser correctement les changements, il est indispensable de les rendre parfaitement comparables, ce qui nécessite une transformation des données initiales. Pour ce faire, il est possible, par exemple, de recourir au carroyage, une méthode cartographique qui consiste à considérer l'occupation du sol à travers une matrice de cellules régulières, i.e. une grille composés de carrés (Antoni, 2002). L'utilisation du sol sur le terrain recouvert par cette grille est alors visualisée à l'intérieur de chacune des cellules, que l'on considère " bâtie ${ }^{12}$ » ou « non bâtie ». Toutefois, afin de mieux caractériser l'étalement urbain et ses liens avec l'expansion démographique ou industrielle et la consommation d'espace $C$ qui en résulte, il apparaît intéressant de distinguer à partir de ces sources d'informations :

$28 C(a)$ : l'ensemble des surfaces bâties; $C(b)$ : l'ensemble des surfaces bâties résidentielles ${ }^{13} ; C(c):$ l'ensemble des surfaces bâties résidentielles équipées ${ }^{14}$. 
29 Ici, nous utilisons des cellules de 50 mètres de côté, afin de prendre en compte avec précision l'évolution des nouveaux logements créés, majoritairement construits sous la forme de maisons individuelles ou de lotissements. En comparant les cellules de date à date, il est possible de dénombrer le nombre de cellules qui ont évolué, et ainsi de calculer la surface construite dans l'intervalle, soit la surface S permettant d'évaluer la consommation d'espace. Considérée en nombre d'hectares, cette surface n'a pas d'autre valeur que celle d'une approximation relativement grossière, dans la mesure où chaque construction, indépendamment de sa taille, est automatiquement assimilée à la surface d'une cellule de 50 mètres de coté, soit 0,25 hectare $^{15}$. De manière relative par contre, cette approximation n'introduit qu'un biais léger qui n'empêche pas les comparaisons dans le temps et de ville à ville.

\section{B. Mesures comparées de la consommation d'espace}

Pour comparer la consommation d'espace dans des secteurs urbains très différents, les données mobilisées s'attachent ainsi à décrire l'expansion spatiale des agglomérations à travers l'analyse de la forme que dessine la surface bâtie. Toutefois si l'étalement touche nettement les trois villes, l'analyse révèle des disparités notables qui répondent à l'histoire démographique des aires urbaines.

31 Morphologiquement d'abord (numérateur S), l'évolution de l'étendue des trois agglomérations apparaît très inégale, et peut être analysée visuellement (malgré l'utilisation d'échelles différentes pour les besoins de représentation cartographique) (Figure 2). Alors que Besançon connaît une expansion remarquable (Figure 2a), Belfort semble un peu moins touchée (Figure 2b), tout comme Montbéliard (Figure 2c) où l'évolution de la tache urbaine est plus difficile à percevoir (ce qui s'explique peut-être aussi par la période d'observation plus courte, 1978-2002). L'analyse quantitative des données confirme l'existence d'écarts importants concernant l'expansion spatiale de chacune des trois agglomérations : l'espace bâti à Besançon est en effet triplé entre 1960 et 2000, alors qu'il est multiplié par 1,6 à Belfort entre 1955 et 1995 et par 1,2 à Montbéliard entre 1978 et 2002. Toutefois, ces résultats peuvent difficilement être mis en comparaison compte tenu des écarts importants entre les différentes périodes d'observation (écarts liés au problème du jeu de données de départ, $c f$. III.A). Afin de pouvoir comparer entre elles chacune des trois agglomérations, il est nécessaire d'établir un taux d'évolution annuel de la croissance morphologique. Ainsi, pour leur période d'observation respective, on constate que Besançon s'est accrue de près de 39 ha par an, alors que Belfort ne gagne que 12,5 ha par an et Montbéliard près de 14,5 ha par an. 
Figure 2 : L'expansion spatiale des trois agglomérations apparaît très inégale : Besançon connaît une expansion remarquable, alors que Belfort semble moins touchée, tout comme Montbéliard, où l'évolution de la tache urbaine est plus difficile à percevoir.

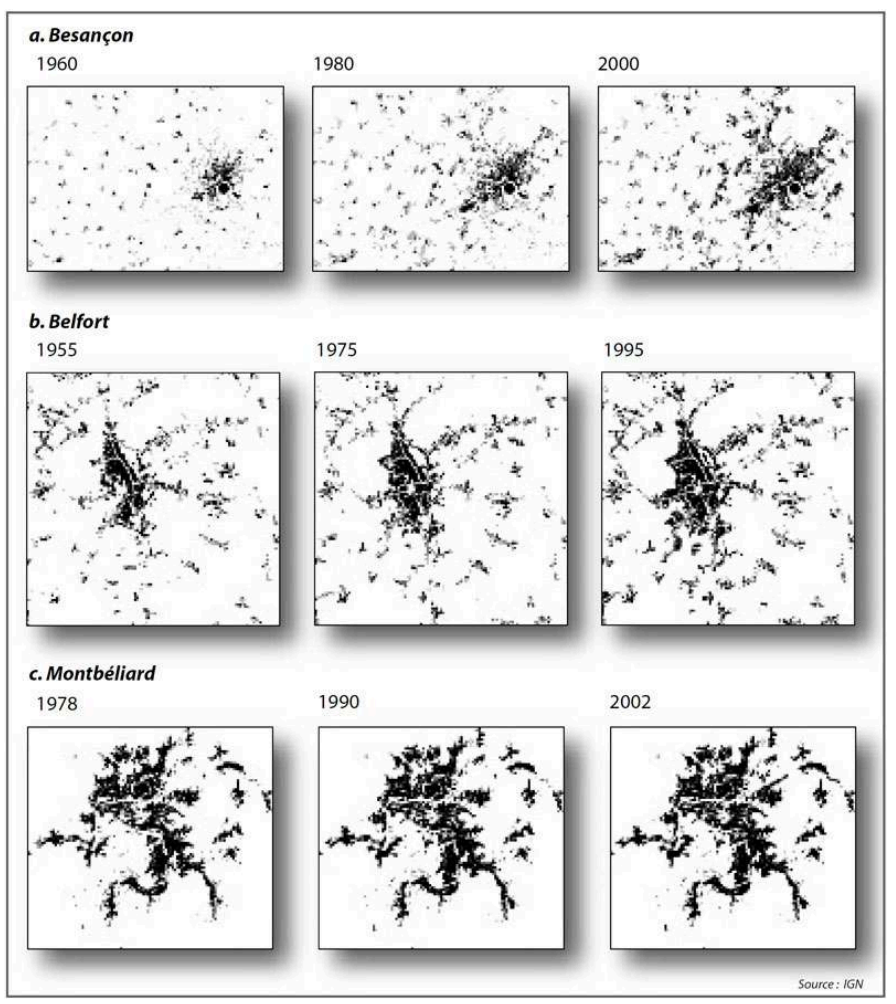

Démographiquement ensuite (dénominateur P), la prise en compte de l'évolution de la population de ces trois villes (réduite aux communes considérées sur le plan morphologique et non plus considérées selon la définition des aires urbaines de l'Insee) permet de mieux caractériser les différents processus d'urbanisation à l'œuvre sur chaque territoire (Figure 3). Dans chacune des trois villes, la période 1955-1975 est marquée par un sensible accroissement de la population. Ainsi, Besançon (Figure 3a) et Belfort (Figure $3 b$ ) connaissent une hausse respectivement de $34 \%$ et $38 \%$ tandis que Montbéliard (Figure 3c) voit sa population augmenter de $85 \%$. Parallèlement, la surface bâtie (notamment résidentielle) s'accroît de façon assez soutenue à Besançon, un peu moins fortement à Belfort (absence de données à Montbéliard). La période 1975-2000 se caractérise ensuite par un ralentissement de l'accroissement de la population, voire une baisse en ce qui concerne Montbéliard. Néanmoins, dans les trois cas, les agglomérations voient leur surface résidentielle bâtie augmenter, de façon assez sensible à Besançon et Belfort. 
Figure 3 : La prise en compte de l'évolution de la population des trois agglomérations permet de mieux caractériser les différents processus d'urbanisation à l'œuvre sur chaque territoire, et offre une perspective pour interpréter l'importance de leur expansion spatiale.
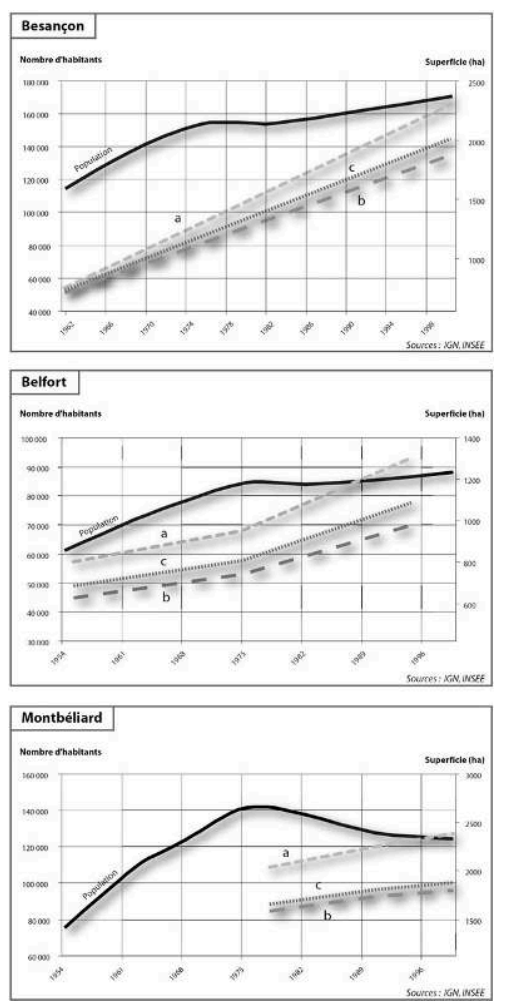

Enfin, la prise en compte simultanée des évolutions démographiques et morphologiques permet d'évaluer la consommation d'espace à l'oeuvre (indicateur $C$ ). Les graphiques ci-dessous (Figure 3) montrent l'évolution de la population couplée à l'évolution de la surface bâtie. Celle-ci se décline en trois courbes qui figurent soit l'ensemble de la surface bâtie $C(a)$ soit la surface résidentielle $C(b)$, soit la surface résidentielle équipée $C(c)$. Pour chaque graphique, l'écart existant entre les courbes (a) et (c) représente la surface occupée par des bâtiments consacrés aux activités professionnelles. À nouveau, des disparités importantes sont à noter: si Belfort et Montbéliard comptent respectivement $16,8 \%$ et $21,5 \%$ de leur surface bâtie dédiée à des zones d'activités autour des années 2000, le taux est sensiblement plus bas à Besançon avec seulement $12,6 \%$. Ceci témoigne de l'importance du secteur industriel dans l'aire urbaine Belfort-Montbéliard où les unités de productions sont relativement consommatrices d'espace (Peugeot à Sochaux et à Montbéliard, Alstom à Belfort). Néanmoins, Besançon comble aujourd'hui son retard et connait depuis les années 1960 une augmentation constante de la surface consacrée aux activités économiques et industrielles.

Finalement, la comparaison de la consommation d'espace entre des villes, issues de contextes différents et à des époques différentes, peut s'appréhender de manière comparative. Pour ce faire, la figure 4 décrit la superficie résidentielle équipée par habitant pour les trois villes à chaque date, ce qui permet de situer chaque résultat à la fois dans l'espace et dans le temps (Figure 4). Bien que Besançon ait connu la plus forte progression de consommation d'espace bâti entre 1960 et 2000, on y lit que chaque habitant y occupe moins d'espace qu'à Belfort et a fortiori à Montbéliard, qui connaît pourtant une baisse de population depuis les années 1975. Globalement, on constate ici 
une hausse généralisée de la consommation d'espace par habitant depuis les années 1950, ce qui confirme les hypothèses posées plus haut à propos de l'étalement urbain, et plus spécifiquement des formes actuelles de l'urbanisation et des processus qui y sont à l'œuvre. On constate ainsi que les citadins sont de plus en plus consommateurs d'espace, ce qui appuie l'idée d'un processus de desserrement des lieux de résidence. La seule exception concerne la ville de Belfort où la superficie bâtie par habitant était plus importante en 1955 qu'en 1975.

Figure 4 : La prise en compte simultanée des évolutions démographiques et morphologiques permet d'évaluer la consommation d'espace, et de constater que, rapportée au nombre d'habitants, elle augmente de manière générale dans chacune des trois villes.

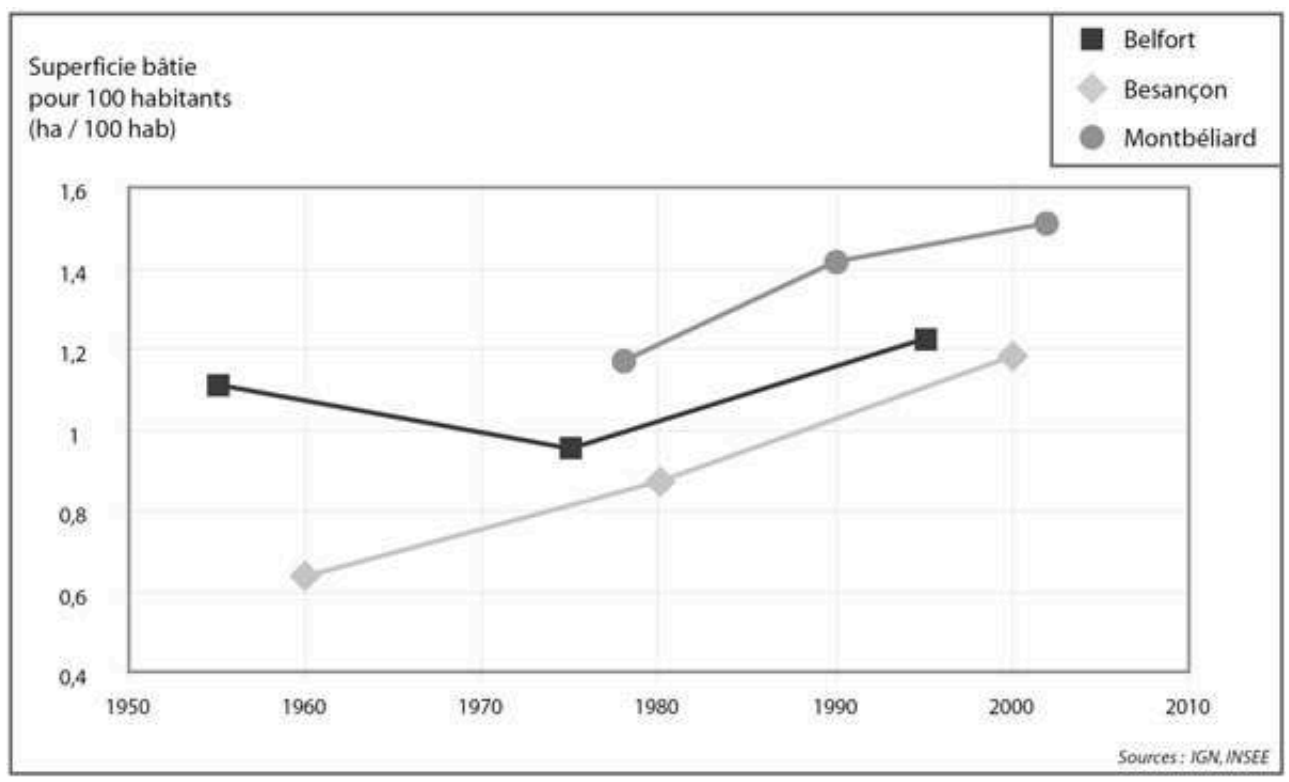

\section{Conclusion}

En conclusion, les chiffres issus de cette étude ont mobilisé des données peu standard et difficiles à comparer, et ont nécessité la mise au point d'un indicateur spécifique. Ils permettent de confirmer l'hypothèse que l'étalement urbain provoque une surconsommation d'espace puisque le nombre d'hectares bâtis rapporté au nombre d'habitants croît globalement de manière constante (sauf l'exception belfortaine). Cette sur-consommation est d'autant plus notable qu'elle apparaît plus ou moins indépendante de la démographie des aires urbaines et accompagne autant la croissance (Besançon) que le décroissance démographique (Montbéliard). Ce paradoxe semble donc bien montrer que l'étalement urbain s'accompagne d'un nouveau mode d'habiter et d'un nouveau rapport à l'espace que les politiques d'urbanisme doivent désormais prendre en compte et anticiper dans le cadre de l'intelligence territoriale (Pascaru, 2006). 


\section{BIBLIOGRAPHIE}

ANTONI J.P. (2002). — « Urban sprawl modelling: a methodological approach ». In : Cybergeo : European Journal of Geography, $\mathrm{n}^{\circ}$ 207, march 2002, 11 p.

ANTONI J.P. (2003). - Modélisation de l'étalement urbain. Aspects conceptuels et gestionnaires, Thèse de doctorat, Strasbourg, Université Louis Pasteur, 535 pages.

BARCELO M. (1993). - « L'étalement urbain, qu'en est-il ? Le définir avant même de tenter de le gérer ». In : Bussière Y., Bonnnafous A. (ss. dir.), Transport et étalement urbain : les enjeux, Programme pluri-annuel en sciences humaines, Rhône-Alpes, p. 29-34.

BARLOGIS O., WIDMER S. (2002). - « Pays de Montbéliard : les départs des jeunes actifs limitent la hausse du chômage ». In : Insee Franche-Comté - L'essentiel, n 52, 4 p.

BARLOGIS O. (2000). - « Aire urbaine de Besançon : une croissance qui s'affirme ». In : Insee Franche-Comté, $\mathrm{n}^{\circ} 21$, mai 2000, pp. 12-14.

BASSAND M., THI NGOC DU T., TARRADELLAS J., BOLAY J.C. (2000). - « Métropolisation, crise écologique et développement durable », Presses polytechniques et universitaires romandes, 312 p.

BRIGAND D., BRIOT L. (2004). — « Maintien de l'emploi qualifié : un enjeu pour Belfort et sa périphérie ». In : Insee Franche-Comté - L'essentiel, n 71, 4 p.

DUSSAUD F.X., MAQUIN I., WIDMER S., BRIGAND D. (2007). — « Les évolutions sociodémographiques récentes dans les trois grandes aires urbaines de Franche-Comté ». In : Insee Franche-Comté - L'essentiel, $\mathrm{n}^{\circ}$ 99, $6 \mathrm{p}$.

FLOCH J.M. (2000). - « Espaces urbains, espaces ruraux : une périurbanisation qui change de visage ». In : Insee Franche-Comté, n²1, mai 2000, pp. 6-9.

FLOCH J.M. (2001). - « Expansion urbaine et densification du peuplement : le cas de Besançon ». In : Insee Franche-Comté - Le Magazine, ${ }^{\circ} 23$, p. 8-10.

FRANKHAUSER P. (1994). - La fractalité des structures urbaines, Economica, 291 p.

FRÉMONT A. (2003). - Portrait de la France. Villes et régions, Flammarion, 782 p.

GRAFMEYER Y., JOSEPH I. (1979). - L'école de Chicago. Naissance de l'écologie urbaine, Ed. du champ urbain, $335 \mathrm{p}$.

HANRIOT E. (2003). - «L'emploi a progressé de 10\% en Franche-Comté entre 1989 et 2000 ». In : Insee Franche-Comté - L'essentiel, $\mathrm{n}^{\circ}$ 58, $6 \mathrm{p}$.

LAURENT J. (2000). - «Aire urbaine de Belfort : sur de bons rails ». In : Insee Franche-Comté, n²1, mai 2000 , pp. $18-20$

LAURENT J. (2000). - « Aire urbaine de Montbéliard : la population continue de baisser ». In : Insee Franche-Comté, n² 21, mai 2000, pp. 15-17.

LE JEANNIC T. (1997). - « Trente ans de périurbanisation : extension et dilution des villes ». In : Économie et statistique, $\mathrm{n}^{\circ}$ 307, p. 21-41.

MERENNE-SCHOUMAKER B. (2002). - La localisation des industries. Enjeux et dynamiques, Presses universitaires de Rennes, $243 \mathrm{p}$. 
MERENNE-SCHOUMAKER B. (2003). - Géographie des services et des commerces, Presses universitaires de Rennes, $240 \mathrm{p}$.

MOINE A. (2001). - « Population et territoire : une incessante recomposition ». In : Images de Franche-Comté, Besançon, ThéMA, p. 6-9.

MONNET J. (1997). - « Pitié pour les grandes villes ! Big Cities Blues : myth or reality?». In : CyberGeo: European Journal of Geography, n 16, février 1997, 3 p.

NAUROY F., SALAMON Y., SARRAZIN D., WIDMER S. (2007). - « La croissance de la population active des zones d'emploi de Belfort et Montbéliard s'accélère depuis 1999 ». In : Insee FrancheComté - L'essentiel, $\mathrm{n}^{\circ} 96,6 \mathrm{p}$.

PASCARU M. (2006). - Intelligence territoriale et gouvernance locale, Cluj-Napoca, Presa Universitara Clujena, $139 \mathrm{p}$.

\section{NOTES}

1. Réalisé par C. Russel, le film The Blob (1988) présente un monstre de forme gélatineuse, visqueuse et répugnante qui ne fait que croître et engloutit tout au fur et à mesure qu'il se développe.

2. Parmi les dictionnaires de géographie les plus courants (Brunet R., Ferras R., Théry H. (2005) Les mots de la géographie - Dictionnaire critique - 3e édition - Reclus - La Documentation Française, Paris - 518 p. ; Lévy J., Lussault M. (2004) - Dictionnaire de la géographie et de l'espace des sociétés - Belin, Paris - 1033 p. ; Baud P., Bourgeat S., Bras C. (1995) - Dictionnaire de géographie - Hatier, Paris - 432 p. ; Johnston R.J., Gregory D., Pratt G., Watts M. (2000) - The dictionary of human geography - 4th edition - Blackwell Publishing, Oxford (UK) - 958 p. ; Merlin P., Choay F. (2000) - Dictionnaire de l'urbanisme et de l'aménagement - 3e édition - Presses universitaires de France, Paris - 902 p. ; Wackermann G. (2005) - Dictionnaire de géographie Ellipses, Paris - 432 p.), seuls les ouvrages de R. Brunet et al. et G. Wackermann (ss. dir.) relaient l'expression « consommation d'espace » en tant que telle. L'expression « Consommation d'espace par les transports » est par contre présente dans le dictionnaire de P. Merlin et F. Choay, mais dans un sens plus spécifique, qui diffère de l'idée présentée ici.

3. La remarque exprimée par R. Brunet (1992) à ce sujet souligne toutefois une subtilité, en insistant sur le fait qu'il n'y a pas véritablement de réduction d'espace, dans la mesure où l'espace est quantitativement toujours le même (il ne parle pas alors d'espace disponible) et ne peut d'ailleurs pas diminuer (la surface terrestre étant toujours égale à elle même). Il note également que la modification de cet état va parfois dans le sens d'une forte valorisation : l'espace urbain étant généralement fonctionnellement plus « productif » que l'espace rural ou en friche, ce qui contredit l'hypothèse d'une dévalorisation qualitative.

4. On ne retrouve aucune ville d'envergure nationale ou internationale semblable à Lyon au sud, Dijon à l'ouest, Nancy-Metz au nord, Strasbourg au nord-est ou au réseau urbain suisse à l'est.

5. L'agglomération est ici entendue au sens de la Communauté d'Agglomération du Grand Besançon (CAGB) soit 59 communes.

6. Parallèlement, la structure du logement semble avoir évolué : la ville connaît une demande croissante de petits logements, qui répond à l'augmentation du nombre de familles monoparentales et au mouvement de décohabitation qui l'accompagne. Alors que 315 logements suffisaient pour abriter 1000 personnes en 1968, il en faut aujourd'hui 430 soit un tiers de plus environ.

7. L'aire urbaine belfortaine (au sens de l'INSEE) se compose de 64 communes, soit 98.000 habitants en 1999. 
8. Les usines de Peugeot constituent le plus grand établissement industriel d'Europe avec près de 20000 emplois se localisant dans la commune de Sochaux, qui constitue une enclave dans Montbéliard.

9. Parallèlement, les mouvements de décohabitation sont importants également dans le Nord de la Franche-Comté : il faut aujourd'hui 58 logements pour loger 100 personnes à Montbéliard contre 52 en 1990.

10. Les fichiers de données ont été mis à disposition par l'Agence de Développement et d'Urbanisme du Pays de Montbéliard (ADUPM).

11. Mais dans les trois cas, le manque de données entre les deux dates contribue à biaiser l'étude du processus d'étalement urbain dans la mesure ou il oblige à « linéariser » son évolution, et à postuler qu'il s'est déroulé de façon constante et identique sur une période de parfois plus de 40 ans.

12. Ces surfaces n'incluent pas l'espace routier (routes, rocades, parkings) associé à la desserte des zones bâties.

13. Les surfaces bâties résidentielles comprennent l'emprise de l'ensemble des bâtiments à destination majoritairement résidentielle, qu'ils soient composés de logements collectifs, denses, ou de maisons individuelles.

14. Les surfaces bâties résidentielles équipées comprennent l'ensemble des surfaces bâties résidentielles auxquelles s'ajoutent les équipements (stades, cimetières, etc.) et les structures d'encadrement (écoles, centres sportifs et socio-culturels, mairies, hôpitaux, etc.).

15. Afin de réduire ce problème, on peut partir du principe que parmi les cellules construites, certaines ne contiennent que très peu de bâti (disons qu'elles sont presque complètement vides), alors que d'autres sont très denses (disons qu'elles sont presque complètement bâties). Si l'on pose l'hypothèse que ces deux extrêmes se compensent, on peut estimer que chaque cellule considérée comme bâtie est moyennement bâtie, et la surface bâtie qu'elle contient réellement peut être approximée à 0,125 ha environ. Le résultat reste approximatif, mais il offre un indicateur intéressant, notamment s'il s'agit de comparer l'évolution simultanée de plusieurs communes ou agglomérations.

\section{RÉSUMÉS}

L'étalement urbain constitue une forme d'urbanisation qui s'est généralisée dans les villes françaises. Portée par une mise en valeur privilégiée des périphéries urbaines, il provoque nécessairement l'accroissement des surfaces bâties de chaque agglomération. Mais dans le même temps, on peut poser l'hypothèse qu'il introduit une sur-consommation d'espace, dont nous formalisons ici une définition. Cette hypothèse est alors testée sur les trois principales agglomérations de Franche-Comté dont les caractéristiques géographiques, démographiques et industrielles très différentes sont brièvement décrites. L'étude confirme globalement la réalité d'une sur-consommation d'espace liée à l'étalement urbain, qui semble globalement indépendante des caractéristiques individuelles de chacune des agglomérations étudiées.

Urban sprawl constitutes a specific case of urbanization that is currently common in French cities. Arising from significant development of suburbs, it inevitably leads to the increase of built-up surfaces in every periurban area. At the same time, we can assume that urban sprawl induces over-consumption of space. This assumption is tested on the three main towns of 
Franche-Comté, that are associated with very different geographical, demographic and industrial characteristics (these characteristics are briefly described). The study confirms, in general, the reality of over-consumption of space, dependant on urban sprawl; it seems generally independent of the individual characteristics of each studied area.

Durch die bevorzugte Entwicklung der städtischen Peripherie nimmt die bebaute Fläche in den Ballungsräumen ständig zu. Es ist davon auszugehen, dass diese Entwicklung zu einem übersteigerten Flächenverbrauch führt. Diese Annahme wird am Beispiel der drei bedeutendsten Städte der Franche-Comté überprüft. Dabei werden deren sehr unterschiedlichen geografischen, demographischen und industriellen Merkmale kurz beschrieben. Die Untersuchung bestätigt insgesamt die Existenz eines durch die Verstädterung bedingten übersteigerten Flächenverbrauchs. Dieser ist kaum von den besonderen Gegebenheiten der verschiedenen Ballungsräume abhängig.

\section{INDEX}

Schlüsselwörter : Flächenverbrauch, Franche-Comté, Stadtentwicklung, Verstädterung Keywords : Franche-Comté, grid mapping, space consumption, Urban sprawl, urbanization Mots-clés : carroyage, consommation d'espace, Étalement urbain, Franche-Comté, urbanisation

\section{AUTEURS}

\section{JEAN-PHILIPPE ANTONI}

Laboratoire ThéMA - CNRS UMR 6940 - Université de Franche-Comté - 32 rue Mégevand, 25000 Besançon - jean-philippe.antoni@univ-fcomte.fr

\section{SAMY YOUSSOUFI}

Laboratoire ThéMA - CNRS UMR 6940 - Université de Franche-Comté - 32 rue Mégevand, 25000 Besançon - samy.youssoufi@univ-fcomte.fr 